\title{
Avaliação da introdução de tecnologias no sistema de produção de gado de corte no Pantanal. Análise de eficiência ${ }^{1}$
}

\section{Urbano Gomes Pinto de Abreu², Paulo Sávio Lopes ${ }^{3}$, Antonio José M. dos Santos Baptista 4 , Robledo de Almeida Torres ${ }^{3}$, Heleno do Nascimento Santos ${ }^{5}$}

\footnotetext{
1 Trabalho desenvolvido com apoio da Coordenação de Aperfeiçoamento de Pessoal de Nível Superior (CAPES).

2 Embrapa Pantanal, Caixa Postal 109, CEP: 79320-900, Corumbá-MS.

${ }^{3}$ Departamento de Zootecnia da Universidade Federal de Viçosa (DZO/UFV).

${ }^{4}$ Doutorando, Departamento de Economia Rural da Universidade Federal de Viçosa (DER/UFV).

${ }^{5}$ Departamento de Informática da Universidade Federal de Viçosa (DPI/UFV).
}

RESUMO - Utilizando-se a metodologia de análise envoltória de dados (DEA), analisou-se, neste estudo, a eficiência de um sistema de produção no qual foram implantadas tecnologias ao longo de oito anos (1995-2002). Foram consideradas informações sobre dez categorias de gastos com insumos (inputs) e uma categoria de produto (output). Utilizou-se a técnica multivariada de componentes principais, com o objetivo de diminuir o número das variáveis de input. Os insumos sal mineral/ vacinas/medicamentos, mão-de-obra/encargos sociais e investimentos apresentaram correlação significativa com os três primeiros componentes principais, que explicaram 91,6\% da variância total. As eficiências verificadas nos anos de 1995, 1996, 1997, 1998, 1999, 2000, 2001 e 2002, respectivamente, foram de 0,$8290 ; 0,6571 ; 1 ; 0,5390 ; 0,7990 ; 0,8010 ; 1$ e 0,9291 , para os modelos de retorno constante à escala (CCR), e de 0,$8841 ; 0,7460 ; 1 ; 0,6350 ; 0,8530 ; 0,9342 ; 1$ e 1 , para retorno variável à escala (BCC). Os anos ineficientes foram decorrentes de receitas crescentes ou decrescentes à escala, ou seja, o produtor gastou insumos com ineficiência, em razão da necessidade de equacionar melhor os gastos com determinados insumos, especialmente do item investimentos.

Palavras-chave: análise envoltória de dados (DEA), bovino de corte, sistema de produção

\section{Evaluation of technology introduction in a beef cattle production system in Pantanal wetlands. Analysis of efficiency}

\begin{abstract}
Data envelopment analysis (DEA) was used to analyze the efficiency of a beef production system in which technologies were adopted during eight years (1995 to 2002). Ten inputs and one output were used to evaluate the production system efficiency applying principal component analysis. The inputs "mineral salt/vaccine/medication", "workforce and social security" and "investment" were significantly correlated with the first three components that explained $91.6 \%$ of the total variance. The efficiencies calculated by DEA for 1995, 1996, 1997, 1998, 1999, 2000, 2001 and 2002 were respectively $0.8290,0.6571,1,0.5390,0.7990,0.8010,1$ and 0.9291 for constant returns to the scale (CCR) models and $0.8841,0.7460$, $1,0.6350,0.8530,0.9342,1$ and 1 for variable returns to scale (BCC) models. The inefficient years were due to increasing returns or decreasing returns to scale suggesting that the rancher did not spend on inputs efficiently. The input "investment" needed to be more adjusted to the other inputs.
\end{abstract}

Key Words: beef cattle, data envelopment analysis (DEA), production system

\section{Introdução}

Apesar da grande deficiência de informação sobre o desempenho da bovinocultura de corte no Brasil, sua importância para o agronegócio é inquestionável. Atualmente, a atividade é responsável por aproximadamente $47 \%$ do total da produção brasileira de carnes, sendo desenvolvida em quase todos os municípios brasileiros, por meio de diferentes sistemas de produção e de grande variabilidade nos níveis de produtividade (Bliska \& Gonçalves, 1998).
O processo de tomada de decisão pelo produtor rural é complexo e, quase sempre, marcado por múltiplos objetivos, alguns que não são de natureza econômica (Cezar et al., 2000). Entretanto, a decisão do pecuarista é sempre guiada pela insatisfação com a situação atual de sua atividade como negócio. Nesse contexto e considerando a crescente demanda, pode-se afirmar que aumentar os desempenhos produtivo e econômico da atividade constitui, hoje, o objetivo mais importante das decisões (Cezar, 2001) para produzir de forma econômica, eficiente e competitiva. Essa 
exigência resulta em intensificação do sistema de produção, mesmo que não seja total e no mesmo nível nas diversas regiões (Euclides Filho, 2000).

A melhoria da eficiência nos sistemas de produção de gado de corte pode ser obtida de diversas formas, entre elas, o desenvolvimento de sistemas especializados, como a especialização na fase de cria. Entretanto, em qualquer situação, a utilização de tecnologia é responsável por incrementos importantes nos índices zootécnicos dos rebanhos (Euclides Filho, 2000).

Em um sistema de produção, identificam-se dois tipos de eficiência: técnica e econômica (Gomes, 1999). Considera-se tecnicamente eficiente quando não há outro sistema que possibilite o mesmo nível de produção ao utilizar quantidade inferior ou igual de insumos. A máxima eficiência econômica ocorre quando não há outro sistema de produção alternativo que produza a mesma quantidade, a menor custo ou com maior lucro. Entretanto, para que um sistema seja economicamente eficiente, é necessário que haja eficiência técnica (Gomes, 1999).

A pecuária de corte, no Pantanal, é desenvolvida em criatórios naturais extensivos, cujas características de manejo são pautadas no regime de enchentes (Pott et al., 1989). Atualmente, há concentração dos produtores do Pantanal na atividade de cria, visto que a recria é feita apenas com novilhas de reposição.

Um indicador do desempenho de determinado sistema produtivo é sua eficiência relativa. Determinar a eficiência de um sistema de produção, identificando-se os motivos, contribui para a definição de estratégias apropriadas para solução do problema. A intenção de corrigir as causas que originaram a ineficiência direciona os planos para melhor aproveitamento dos recursos investidos na introdução e adaptação de tecnologias (Arzubi \& Berbel, 2002).

Neste trabalho, objetivou-se, por meio da análise envoltória de dados (DEA), avaliar a eficiência da introdução de diferentes tecnologias no sistema de produção tradicional de cria extensiva do Pantanal.

O conhecimento da eficiência e a identificação das ineficiências no processo de introdução de tecnologias permitem orientar decisões futuras visando à melhoria da capacidade competitiva das propriedades no Pantanal.

\section{Material e Métodos}

O trabalho foi desenvolvido na fazenda Alegria (retiro Chatelodo), em uma área de 6.364 ha, na sub-região de Nhecolândia, em Mato Grosso do Sul.

O manejo de gado no retiro Chatelodo, onde se efetuou a fase de cria, foi realizado por três peões residentes. A recria de machos e de fêmeas foi feita em outra parte da fazenda. No manejo tradicional, as vacas e os touros não foram identificados e o descarte foi realizado sem maior controle. $\mathrm{O}$ manejo na propriedade resumiu-se aos "trabalhos de gado" anuais, o primeiro em maio ou junho e o segundo em novembro ou dezembro.

O projeto foi desenvolvido em cinco anos (maio de 1994 a maio de 1999) e as tecnologias foram implantadas, paulatinamente, ao longo do trabalho de monitoramento, conforme descrito por Abreu (2004).

À medida que foram introduzidas no sistema de manejo no retiro, as tecnologias eram implantadas em toda a Fazenda Alegria, sob administração direta do proprietário. Todos os custos de implantação (insumos - input) foram registrados, assim como as vendas de animais (produtos - output), de modo que os dados econômicos foram registrados em conjunto, sem separação por unidade da propriedade. Dessa forma, as mudanças implantadas no sistema tradicional de produção foram estendidas a toda a propriedade. A modificação no sistema foi definitiva e os dados econômicos continuaram sendo registrados mesmo após o término do trabalho de monitoramento. Assim, as coletas de dados econômicos foram feitas em oito anos (1995 a 2002).

As despesas com a propriedade foram classificadas, tradicionalmente, em dez itens:

1. sal mineral/vacinas/medicamentos;

2. combustíveis;

3. utensílios/ferramentas para atividade pecuária;

4. gastos com impostos e escritório;

5. manutenção de máquinas e veículos;

6. mão-de-obra e encargos sociais;

7. fretes para a propriedade;

8. investimentos (aquisição de touros, trator, utilitários etc);

9. viagem do proprietário à fazenda; e

10. comissão de vendas (o proprietário sempre comercializava os animais em leilões rurais).

As despesas foram corrigidas pela inflação, de acordo com o índice geral de preços (IGP) fornecido pelo Instituto de Pesquisas Econômicas Aplicadas (IPEA). As receitas são advindas da venda das diferentes categorias de bovinos de corte no Pantanal, descritas por Abreu et al. (2001).

A análise envoltória de dados (DEA) foi originalmente proposta por Charnes et al. (1978), como metodologia para avaliação da eficiência relativa de unidades homogêneas que possuem autonomia na tomada de decisão (Decision Making Units - DMU). Os modelos DEA são baseados em amostra de dados observados em diferentes DMUs, os quais são de insumos e de produtos. Os objetivos eram 
construir um conjunto de referência convexo e classificar as DMUs em eficientes ou ineficientes, adotando-se como referencial a superfície formada. De acordo com as pressuposições específicas de cada modelo, o conjunto-referência teria determinada forma. Nessas pressuposições, estariam contidas as informações a respeito do tipo de retorno à escala. Desde a publicação do trabalho original (Charnes et al., 1978), DEA tornou-se muito utilizada na análise da eficiência de vários tipos de organizações e situações (Gomes, 1999).

O modelo desenvolvido por Charnes et al. (1978) é chamado modelo com retornos constantes à escala (CCR) e foi reformulado por Banker et al. (1984), com o objetivo de possibilitar a análise no caso de retornos variáveis à escala (BCC).

$\mathrm{Na}$ análise em que se utilizou DEA, formulou-se o modelo a ser utilizado, cuja resolução determinou a fronteira de produção como um envoltório nos dados, definindo, em cada DMU, se ela está ou não na fronteira de eficiência. A utilização da metodologia não requer especificação de forma funcional que relacione os dados, por ser uma técnica não-paramétrica, o que permite a análise de retornos variáveis à escala $(\mathrm{BCC})$ e a quantificação de eficiência da escala (Arzubi \& Berbel, 2002).

DEA é uma técnica baseada em programação linear que visa à mensuração do desempenho relativo de unidades organizacionais em que ocorre presença de múltiplos insumos e produtos relativamente homogêneos. No caso simples, em que o processo de produção possui apenas um insumo e apenas um produto, a eficiência pode ser definida por:

\section{Eficiência $=$ Produto/Insumo}

De forma geral, as organizações possuem grande número de entradas e de saídas, complexidade que pode ser incorporada na mensuração da eficiência, pela seguinte definição:

\section{Eficiência $=$ Soma ponderada dos produtos $/$ Soma ponderada dos insumos}

Essa definição requer uma série de pesos difíceis de serem estimados, particularmente se uma série de pesos iguais for aplicada a grande número de unidades organizacionais com diferentes características. Esse problema pode ser resolvido pela definição de que cada unidade individual pode possuir seu próprio sistema de valores e, conseqüentemente, definir seu próprio sistema de pesos para ponderação (Boussofiane et al., 1991).

Charnes et al. (1978) propuseram que a eficiência da unidade $j_{0}$ poderia ser avaliada pela solução do seguinte modelo:
$\operatorname{Max} h_{0}=\frac{\sum_{r=1}^{t} u_{r} y_{r j 0}}{\sum_{i=1}^{m} v_{i} x_{i j 0}}$

sujeito a:

$\frac{\sum_{r=1}^{t} u_{r} y_{r j}}{\sum_{i=1}^{m} v_{i} x_{i j}} \leq 1, \quad j=1, \ldots, n$,

$u_{r}, v_{i} \geq \varepsilon, \quad \forall \quad r$ e $i$,

em que $y_{r j}=$ total de produtos $r$, oriundo da unidade produtiva $j, x_{i j}=$ total de insumos $i$, proveniente da unidade produtiva $j ; u_{r}=$ peso dado aos insumos $r ; v_{i}=$ peso dado aos produtos $i ; n=$ número de unidades produtivas; $t=$ número de produtos; $m=$ número de insumos; $\mathrm{e}$ $\varepsilon=$ número pequeno e positivo (tolerância).

Na solução desse modelo, a eficiência da unidade $j_{0}$ é maximizada, sujeita à restrição de que as medidas de eficiência de todas as outras unidades produtivas sejam menores ou iguais a 1. A principal característica desse modelo é que os pesos $u_{r}$ e $v_{i}$ são tratados como incógnitas e foram escolhidos para que a eficiência de $j_{0}$ fosse maximizada. Se a eficiência de $j_{0}$ é igual a 1 , é considerada eficiente em relação às demais unidades produtivas; caso contrário, é ineficiente. No caso de uma unidade ser ineficiente, a solução identificaria unidades eficientes correspondentes que formam um grupo de referência benchmark) para unidades ineficientes (Boussofiane et al., 1991).

Atualmente, para calcular a eficiência relativa de uma DMU, resolve-se o problema dual, modelo que pode ser descrito no caso de retornos constantes à escala (CCR), de acordo com as notações de Coelli (1996) e Arzubi \& Berbel (2002), da seguinte forma:

$$
\begin{aligned}
& \operatorname{Min}_{\theta, \lambda} \theta \\
& \text { sujeito a: } \\
& -y+Y \lambda \geq 0 \\
& \theta x_{i}-X \lambda \geq 0 \\
& \lambda \geq 0
\end{aligned}
$$

em que $y=$ produto da DMU sob análise; $x=$ insumo da DMU sob análise; $X=$ matriz de insumos $\mathrm{K} \times \mathrm{N} ; Y=$ matriz de produtos $\mathrm{M} \times \mathrm{N} ; \theta=$ uma escalar que multiplica os vetores 
dos insumos; $\lambda=$ vetor $\mathrm{N}$ x 1 de constantes que multiplica a matriz de insumos e produtos; e $N=$ número de DMUs.

O modelo dual permite a análise da eficiência relativa, fornecida nos casos em que existam folgas (slacks) ou reduções não-radiais nos insumos. Para que uma DMU seja considerada tecnicamente eficiente, $\theta$ deve ser igual a $1 \mathrm{e}$ as folgas, iguais a zero. O problema linear deve ser resolvido $\mathrm{N}$ vezes, obtendo-se, para cada iteração, a eficiência relativa de cada DMU.

Banker et al. (1984) desenvolveram importante extensão do modelo DEA com retornos constantes à escala (CCR). Esses autores modificaram o modelo linear, de modo a incorporar restrição de convexidade $\left(N 1^{\prime} \lambda=1\right)$, o que permitiu a análise de receitas variáveis à escala (BCC):

$\operatorname{Min}{ }_{\theta, \lambda} \theta$,

sujeito a:

$-y+Y \lambda \geq 0$

$\theta x_{i}-X \lambda \geq 0$

$N 1^{\prime} \lambda=1$

$\lambda \geq 0$

em que $N 1=$ vetor unitário.

Essa modificação permitiu a decomposição da eficiência técnica em duas: pura e de escala. Para isso, deve-se resolver o problema linear por meio dos dois modelos (M2 e M3) descritos; se houver diferença entre as duas soluções em uma DMU em particular, a DMU possuirá ineficiência de escala e o valor da ineficiência será a razão entre os valores encontrados nos modelos CCR (M2) e BCC (M3).

A eficiência técnica pura coincide com a solução do modelo BCC e a ineficiência de escala origina-se da produção em nível deficiente de escala. O nível ótimo de escala é obtido por meio das comparações com as DMUs eficientes $(\mathrm{CCR}=1)$. A eficiência técnica global é produto das duas eficiências (técnica pura e escala) e sua medição coincide com o modelo CCR.

O modelo DEA pode ter duas orientações, com vistas a otimizar a combinação de insumos (modelo insumo orientado) para produção de produtos, ou otimizar a produção de produtos (modelo produto orientado); diferenças e detalhes da metodologia foram descritos por Coelli (1996) e Gomes (1999).

A interpretação das ineficiências depende do modelo, insumo ou produto orientado. O modelo permite conhecer a proporção de insumos que foi transformada em produtos nas diferentes DMUs, enquanto o insumo possibilita inferir o quanto de produto foi incrementado em todas as DMUs eficientes (Arzubi \& Berbel, 2002). Um indicador interessante que visa diferenciar a ineficiência de escala de uma DMU poderá ser calculado para verificar se a DMU está operando com retornos decrescentes ( $d r s)$ ou em uma área de retornos crescentes em escala (irs). Para isso, deve-se substituir a restrição $N 1^{\prime} \lambda=1$ por $N 1^{\prime} \lambda \leq 1$. Dessa forma, incorpora-se nas restrições a impossibilidade de receitas crescentes à escala; se o novo valor obtido, ao executar a análise, for igual ao obtido no modelo BCC, a DMU estará operando no setor das curvas de receitas decrescentes à escala; caso contrário, a DMU estará operando no setor de receitas crescentes à escala. Logicamente, as DMUs nas quais os resultados dos modelos BCC e CCR são iguais possuem escala ótima e não são consideradas nesta classificação.

Os dados econômicos de cada ano (1995-2002), levantados in loco com o proprietário, foram considerados em uma DMU, pois, ao longo do tempo, as tecnologias foram paulatinamente introduzidas no sistema extensivo tradicional da região (Abreu et al., 2004), sendo o manejo ajustado às características de comercialização, de mão-de-obra e de ambiente do Pantanal. Foi utilizado o programa DEAP, versão 2.1 (Coelli, 1996), na resolução dos problemas lineares da análise DEA.

Tendo-se em vista que as tecnologias foram desenvolvidas visando ao aumento da produção, utilizou-se o modelo de DEA multiestágio de programação, orientação produto com retornos constantes à escala $(\mathrm{CCR})$ e com retornos variáveis à escala (BCC), como descrito por Coelli (1996) e Gomes (1999).

A escolha das quantidades dos insumos e produtos pode afetar a discriminação das DMUs, em relação à análise de eficiência (Boussofiane et al., 1991). A soma do número de produtos e insumos deve ser menor (comparativamente) que o número de DMU, em razão da flexibilidade na escolha dos pesos para determinação da eficiência de cada DMU, individualmente. Na procura da solução eficiente, as DMU podem ser alocadas, quase todas, em um único insumo e produto, no caso de as variáveis escolhidas possuírem correlação alta, não havendo discriminação de sua eficiência. A DMU com alta razão de insumo/produto pode atribuir sua ponderação a todos os pesos de todas as DMU, o que resulta no surgimento de grande número de DMU eficientes. Geralmente, é aplicada a seguinte expressão para verificar o total de número de insumos e produtos: $(m+s)<N / 3$, em que $m=$ número de insumos; $s=$ número de produtos; e $N=$ número de DMU (Friedman \& Sinuany-Stern, 1998).

A técnica multivariada de componentes principais (CP), por meio da análise da estrutura de (co)variância de uma série de variáveis, estima combinações lineares destas 
variáveis, com o objetivo de reduzir a quantidade e a dimensionalidade de dados, além de facilitar a interpretação das relações entre as diferentes variáveis (Johnson \& Wichern, 1999). Componente principal é uma das técnicas de análise exploratória multivariada mais utilizadas (Khattree $\&$ Naik, 2000), sendo empregada nas mais diferentes situações e áreas da pesquisa em produção animal (McManus \& Saueressig, 1998; Cardoso et al., 2003; Azevêdo et al., 2003; Pinto et al., 2005).

Com o objetivo de diminuir o número de insumos na análise de DEA, foi utilizada a técnica de componentes principais. Com os componentes principais que explicaram $90 \%$ da variação total das informações, foi calculada a correlação univariada entre as variáveis e os componentes. As variáveis cujas estimativas tiveram correlações altamente significativas $(\mathrm{P}<0,01)$ com os componentes foram utilizadas como variáveis de insumo na análise de DEA. Utilizaram-se os procedimentos PRINCOMP e CORR na análise de componentes principais e de correlação, do pacote estatístico $S A S$ (SAS, 1999).

\section{Resultados e Discussão}

Os percentuais dos itens de despesas, em relação aos totais dos gastos ao longo dos anos, são apresentados na Tabela 1 .

No sistema de criação analisado, o item "custo da mão-de-obra e encargos sociais" foi o de maior importância relativa para o custo total no período analisado.

Na Tabela 2, observam-se os três componentes principais (CPs) e os respectivos autovalores, que explicaram $91,6 \%$ da variância total do conjunto total de dados. Os autovalores do primeiro, do segundo e do terceiro componentes explicaram 52,$9 ; 28,8$ e $9,8 \%$, respectivamente, da variância total. Os coeficientes das variáveis mão-de-obra/ encargos sociais e investimentos foram os que tiveram maior peso no primeiro e no segundo componente principal, respectivamente, itens que mais influenciaram os componentes principais.

A mão-de-obra é considerada um dos pontos de estrangulamento na melhoria da eficiência produtiva na região (Cadavid Garcia, 1986). A introdução de tecnologias na fazenda promoveu a diminuição da rotatividade na mão-de-

Tabela 2 - Três primeiros componentes principais de insumos e autovalores

Table 2 - $\quad$ First three principal components of inputs and eigenvalues

\begin{tabular}{lccc}
\hline $\begin{array}{l}\text { Autovalor } \\
\text { Eigenvalues } \\
\text { Diferença }\end{array}$ & 193550825,00 & 105485069,00 & 35918032,00 \\
$\begin{array}{l}\text { Difference } \\
\text { Proporção }\end{array}$ & 88065756,00 & 69567037,00 & - \\
$\begin{array}{l}\text { Proportion } \\
\text { Propor. acumulada } \\
\text { Cumulative proportion }\end{array}$ & 0,529 & 0,2885 & 0,0982 \\
\hline Variável & $\mathrm{CP} 1$ & 0,817 & 0,916 \\
Input & & $\mathrm{CP} 2$ & $\mathrm{CP} 3$ \\
\hline $\mathrm{S} / \mathrm{V} / \mathrm{M}$ & 0,3263 & $-0,0238$ & $-0,1553$ \\
$\mathrm{CO}$ & 0,0963 & $-0,0017$ & $-0,0651$ \\
$\mathrm{U} / \mathrm{F}$ & 0,0278 & 0,0994 & $-0,1025$ \\
$\mathrm{I} / \mathrm{E}$ & 0,2689 & 0,1515 & $-0,5934$ \\
$\mathrm{M}$ & 0,2239 & 0,1081 & 0,4629 \\
$\mathrm{MO} / \mathrm{ES}$ & 0,8373 & 0,1976 & 0,1220 \\
$\mathrm{~F}$ & 0,0043 & 0,0548 & $-0,0410$ \\
$\mathrm{I}$ & $-0,2135$ & 0,8999 & 0,2261 \\
$\mathrm{~V}$ & 0,0107 & $-0,0758$ & 0,0113 \\
$\mathrm{CV}$ & 0,1110 & $-0,3119$ & 0,5716 \\
\hline
\end{tabular}

$\mathrm{CP}$ - Componente principal; S/V/M - sal mineral/vacinas/medicamentos; CO - combustíveis; U/F - utensílios/ferramentas; I/E - impostos e escritório; M - manutenção de máquinas; MO/ES - mão-de-obra e encargos sociais; F - fretes; I - investimentos; V - viagem do proprietário para a fazenda; CV - comissão de vendas.

CP - Principal component; S/V/M - mineral salt/vaccines/medications, CO - Fuel; U/F utensils/tools; //E - tax and office expenses; $M$ - machinery and vehicle maintenance; $M O /$ ES - labor and social security taxes; F- hired truck; I - investments (acquisition of bulls tractors, facilities etc); $V$-farmer tripsto the farm; $\mathrm{CV}$ - sales commission.

Tabela 1 - Percentuais dos itens de despesas, em relação ao total de despesa, no período de 1995 a 2002, da fazenda Alegria Table 1 - Percents of expenditure on inputs, in relation to total costs between 1995 and 2002, Alegria farm

\begin{tabular}{|c|c|c|c|c|c|c|c|c|}
\hline $\begin{array}{l}\text { Variável } \\
\text { Input }\end{array}$ & 1995 & 1996 & 1997 & 1998 & 1999 & 2000 & 2001 & 2002 \\
\hline $\mathrm{S} / \mathrm{V} / \mathrm{M}$ & 10,04 & 12,22 & 15,72 & 10,78 & 12,49 & 13,74 & 12,14 & 11,10 \\
\hline $\mathrm{U} / \mathrm{F}$ & 2,63 & 4,33 & 2,46 & 3,18 & 2,95 & 2,34 & 2,65 & 3,33 \\
\hline $\mathrm{I} / \mathrm{E}$ & 5,59 & 14,30 & 10,29 & 8,48 & 7,49 & 9,11 & 10,69 & 6,50 \\
\hline M & 17,01 & 8,96 & 12,61 & 11,74 & 6,93 & 11,18 & 8,70 & 9,64 \\
\hline $\mathrm{MO} / \mathrm{ES}$ & 28,22 & 30,91 & 34,47 & 31,73 & 29,76 & 36,21 & 31,26 & 30,59 \\
\hline V & 3,77 & 2,47 & 2,84 & 1,28 & 1,82 & 2,61 & 3,87 & 3,33 \\
\hline $\mathrm{CV}$ & 6,71 & 0,40 & 5,87 & 5,45 & 7,28 & 9,27 & 12,22 & 9,71 \\
\hline TOTAL & 100 & 100 & 100 & 100 & 100 & 100 & 100 & 100 \\
\hline
\end{tabular}

S/V/M - sal mineral/vacinas/medicamentos; CO - combustíveis; U/F - utensílios/ferramentas; I/E - impostos e escritório; M - manutenção de máquinas; MO/ ES - mão-de-obra e encargos sociais; F - fretes; I - investimentos; V - viagem do proprietário para a fazenda; CV- comissão de vendas.

S/V/M - mineral salt/vaccines/medications, CO - Fuel; U/F - utensils/tools; I/E - tax and office expenses; $M$ - machinery and vehicle maintenance; MO/ES - labor and social security taxes, $F$ - hired truck; I - investments (acquisition of bulls, tractors, facilities, etc.); $V$-farmer trips to the farm; CV - sales commission. 
obra, visando manter, na propriedade, os peões que melhor se adaptaram ao sistema de coleta de informações e ao manejo dos rebanhos de cria, de forma diferente da tradicionalmente realizada na região.

Os itens de investimentos diretamente ligados à introdução de tecnologias foram reforma de cercas e construção de poços de draga.

As correlações dos itens mão-de-obra/encargos sociais (MO/ES), investimentos (I) e sal mineral/vacinas/medicamentos ( $\mathrm{S} / \mathrm{V} / \mathrm{M})$ com os dois primeiros componentes principais foram altamente significativas $(\mathrm{P}<0,01)$ (Tabela 3 ), sendo $\mathrm{S} / \mathrm{V} / \mathrm{M}$ e $\mathrm{MO} / \mathrm{ES}$ correlacionados ao primeiro e investimentos ( I ), ao segundo componente.

Os itens $\mathrm{S} / \mathrm{V} / \mathrm{M}, \mathrm{MO} / \mathrm{ES}$ e I foram considerados itens de insumo na análise DEA, pois as correlações de Pearson entre os itens $\mathrm{S} / \mathrm{V} / \mathrm{M}$ e $\mathrm{MO} / \mathrm{ES}$ com o primeiro componente principal, que explicou aproximadamente $53 \%$ da variância total das informações, foram significativos $(\mathrm{P}<0,01)$ e estimados em 0,9466 e 0,9802 , respectivamente. O item I, cuja correlação estimada foi de 0,9366 com o segundo componente principal, que explicou aproximadamente 30\% da variância total, também foi significativo $(\mathrm{P}<0,01)$.

O estudo da eficiência da implantação das tecnologias no sistema de produção, em termos de unidade monetária de insumos e produto, realizado ao longo do tempo (oito anos), na fazenda Alegria, é sumarizado na Tabela 4. A tendência, ao longo dos anos de implantação, foi de aumentos gradativos na eficiência, tanto no modelo de retornos constantes à escala como no de retornos variáveis à escala.

Consta na Figura 1 a tendência das eficiências, nos modelos CCR e BBC, ao longo do período analisado, de acordo com a visualização dos resultados sugeridos para análise DEA por El-Mahgary \& Lahdelma (1995).
No resultado da metodologia DEA de retornos constantes à escala (CCR), a eficiência técnica média foi de 0,82 e a eficiência média encontrada, de 0,88 . Pelo modelo CCR, os mesmos anos considerados ineficientes também o foram pelo modelo BBC; ou seja, os resultados foram semelhantes nos dois modelos utilizados.

Ocorreu ineficiência técnica durante a implantação das tecnologias nos anos de 1996, 1998 e 2000; ou seja, ocorreram gastos com insumos que poderiam ser reduzidos sem comprometimento do produto (receita com as vendas de bovinos). Nos anos de 1995, 1999 e 2002, os gastos com insumos poderiam ter sido aumentados, pois a receita foi maior. O percentual médio observado no período de 1995 a $2002 \mathrm{em}$ relação ao gasto no insumo I foi de 14,69\%. Nos anos de 1995 , 1996, 1998, 1999 e 2002, os percentuais com gasto em I foram, respectivamente, 19,$35 ; 17,95 ; 18,45 ; 22,32$ e $16,04 \%$, todos maiores que o percentual médio. Os anos de 1997 e 2001 foram considerados eficientes, e o item I representou, respectivamente, 5,23 e 10,23\% do total dos gastos com insumos. No ano de 2000, o item de ineficiência mais significativo foi o gasto

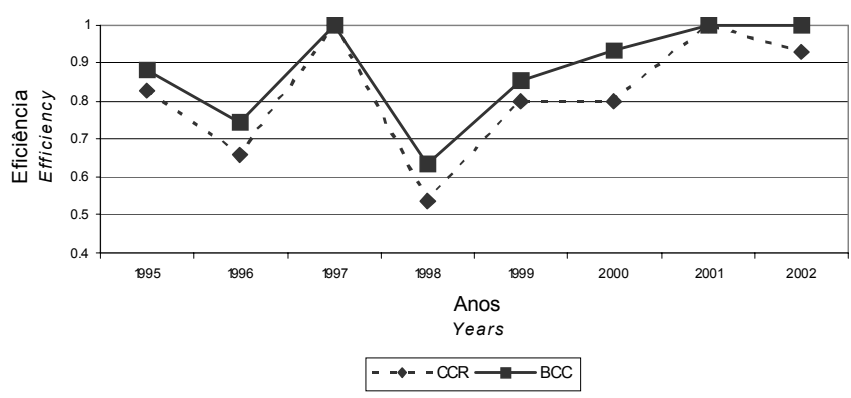

Figura 1 - Eficiência estimada pelos modelos de retornos constantes à escala (CCR) e de retornos variáveis à escala (BCC) no período de acompanhamento.

Figure 1 - Estimates of efficiencies by means of models of constant returns (CCR) and variable returns (BCC) in the period 1995/ 2002

Tabela 3 - Correlações dos itens de insumos com os três primeiros componentes principais

Table 3 - Estimates of correlation between inputs, output and the first three principal components

\begin{tabular}{|c|c|c|c|}
\hline $\begin{array}{l}\text { Variável } \\
\text { Input }\end{array}$ & $\begin{array}{c}\text { CP1 (estimativa da probabilidade) } \\
(p \text {-value })\end{array}$ & $\begin{array}{c}\text { CP2 (estimativa da probabilidade) } \\
(p \text {-value })\end{array}$ & $\begin{array}{c}\text { CP3 (estimativa da probabilidade) } \\
(p \text {-value })\end{array}$ \\
\hline $\mathrm{S} / \mathrm{V} / \mathrm{M}$ & $0,9466(0,0004)$ & $-0,0511(0,9043)$ & $-0,1941 \quad(0,6451)$ \\
\hline $\mathrm{CO}$ & $0,7848(0,0211)$ & $-0,0101(0,9811)$ & $-0,2287(0,5858)$ \\
\hline $\mathrm{U} / \mathrm{F}$ & $0,2921(0,4826)$ & $0,7701(0,0254)$ & $-0,4631(0,2478)$ \\
\hline $\mathrm{I} / \mathrm{E}$ & $0,6660(0,0714)$ & $0,2770(0,5066)$ & $-0,6332(0,0919)$ \\
\hline M & $0,5463(0,1613)$ & $0,1948(0,6439)$ & $0,4866 \quad(0,2214)$ \\
\hline $\mathrm{MO} / \mathrm{ES}$ & $0,9802(<0,0001)$ & $0,1707(0,6860)$ & $0,0615(0,8849)$ \\
\hline $\mathrm{F}$ & $0,4716(0,2381)$ & $0,4427(0,2720)$ & $-0,1932 \quad(0,6466)$ \\
\hline I & $-0,3010(0,4688)$ & $0,9366(0,0006)$ & $0,1373 \quad(0,7457)$ \\
\hline V & $0,1214(0,7746)$ & $-0,6349(0,0908)$ & $0,0551 \quad(0,8969)$ \\
\hline $\mathrm{CV}$ & $0,2723(0,5154)$ & $-0,5646(0,1448)$ & $0,6037(0,1130)$ \\
\hline
\end{tabular}

CP - Componente principal; S/V/M - sal mineral/vacinas/medicamentos; CO - combustíveis; U/F - utensílios/ferramentas; I/E - impostos e escritório; M manutenção de máquinas; MO/ES - mão-de-obra e encargos sociais; F - fretes; I - investimentos; V - viagem do proprietário para a fazenda; CV- comissão de vendas.

CP-Principal component; S/V/M - mineral salt/vaccines /medications, CO - Fuel; U/F - utensils/tools; I/E - tax and office expenses; M - machinery and vehicle maintenance; MO/ES - labor and social security taxes; $F$ - hired truck; I - investments (acquisition of bulls, tractors, facilities, etc.); $V$ - farmer trips to the farm; CV - sales commission. 
Tabela 4 - Estimativas das eficiências por meio dos modelos de retornos constantes (CCR) e variáveis $(\mathrm{BCC})$ à escala, no período de 1995 a 2002, na fazenda Alegria

Table 4 - Estimates of efficiencies by means of models of constant returns $(C C R)$ and variable returns $(B C C)$ to the scale between 1995 and 2002, at Alegria farm

\begin{tabular}{lcccc}
\hline $\begin{array}{l}\text { Ano } \\
\text { Year }\end{array}$ & CCR & BCC & $\begin{array}{c}\text { Escala CCR/BCC } \\
\text { ScaleCCR/BCC }\end{array}$ & $\begin{array}{c}\text { Ineficiência } \\
\text { de escala } \\
\text { Scale inefficiency }\end{array}$ \\
\hline 1995 & 0,8290 & 0,8841 & 0,9381 & irs \\
1996 & 0,6571 & 0,7460 & 0,8800 & $\mathrm{drs}$ \\
1997 & 1,0000 & 1,0000 & 1,0000 & - \\
1998 & 0,5390 & 0,6350 & 0,8480 & $\mathrm{drs}$ \\
1999 & 0,7990 & 0,8530 & 0,9361 & irs \\
2000 & 0,8010 & 0,9342 & 0,8581 & $\mathrm{drs}$ \\
2001 & 1,0000 & 1,0000 & 1,0000 & - \\
2002 & 0,9291 & 1,0000 & 0,9291 & $\mathrm{irs}$ \\
Média & 0,82 & 0,88 & 0,90 & \\
Average & & & & \\
\hline
\end{tabular}

drs - receitas decrescentes à escala; irs - receitas crescentes à escala. drs-decreasing returns-to-scale; irs - increasing returns-to-scale.

excessivo com $\mathrm{MO} / \mathrm{ES}$, que representou $36,21 \%$ do total dos insumos, sendo $31,64 \%$ o percentual médio observado no período avaliado com gasto no insumo $\mathrm{MO} / \mathrm{ES}$.

Os resultados indicam que, nos anos de 1997 e 2001, não houve problema de escala. Uma condição para que o ano apresente máxima eficiência técnica, no modelo CCR, é que sua eficiência técnica, quando na análise no modelo BCC, seja também máxima. Portanto, esses anos citados podem ser considerados de máxima eficiência técnica (eficiência $=1$ ).

As ineficiências de escala foram consideradas do tipo de retornos crescentes (irs) e decrescentes ( $d r s)$ à escala nos anos de 1995, 1999 e 2002 e de 1996, 1998 e 2000, respectivamente. Segundo Gomes (1999), a interpretação de variações na escala de produção como tentativa de aumentar a eficiência técnica não considera as mudanças tecnológicas na atividade do produtor; ou seja, variações no volume de produção (produtividade) podem ser acompanhadas por mudanças tecnológicas.

A produtividade de uma DMU é a relação entre as quantidades de seus produtos e de seus insumos, de modo que a variação é resultado das diferenças na tecnologia, na eficiência dos processos de produção e no ambiente em que ocorre a produção. A eficiência de uma DMU resulta da comparação entre valores observados e valores ótimos de insumos e produtos. Esta comparação assume forma de relação entre a quantidade do produto obtido e seu nível máximo, considerando a quantidade do insumo utilizado ou a relação da quantidade de insumo utilizada e seu mínimo requerido para produzir, considerando a quantidade de produto obtido ou alguma combinação dos dois. Nesta situação, o ótimo é definido em termos de possibilidades de produção e diz respeito à eficiência técnica (Tupy \& Yamaguchi, 1998).
Na Figura 2, observa-se a eficiência estimada pelo modelo CCR, em relação ao fator individual de produto (receita anual). A média e o desvio-padrão no período da receita anual foram calculados em 305.162,26 $\pm 50.128,03$ reais, embora a eficiência máxima no ano 2001, não necessariamente a maior receita, tenha resultado diretamente em maior eficiência. Por exemplo, a eficiência no ano de 1997 foi estimada em 1, enquanto, no ano 2000, obteve-se receita anual maior. No ano 2000, no entanto, o gasto de $36,21 \%$ com o insumo MO/ES (36,21\%), em relação ao total dos gastos, foi o maior no período analisado.

As receitas alcançadas em 1995, 1996, 1997, 1998, 1999 e 2002 podem ser consideradas semelhantes, mesmo que apenas o ano de 1997 tenha sido considerado de eficiência máxima, provavelmente em razão do pequeno percentual $(5,23 \%)$ de gasto com o item de insumo I. Possivelmente, a modificação tecnológica por que passava o sistema de produção, com ajustes realizados durante o período, tenha sido o motivo de os gastos em insumos serem proporcionalmente diferentes no período, especialmente na categoria de investimentos.

Nas Figuras 3, 4 e 5, pode-se visualizar a eficiência no período em relação às três categorias de insumos: sal mineral/vacinas/medicamentos $(\mathrm{S} / \mathrm{V} / \mathrm{M})$, mão-de-obra e encargos sociais (MO/ES) e investimentos (INV).

O gasto médio e o desvio-padrão, no período avaliado, com o insumo S/M/V foram de 20.015,96 $\pm 4.795,51$ reais. Em 2001, o gasto foi menor que a média com esse tipo de insumo, uma das características que contribuíram para maior eficiência. O ano de 1997 foi um dos que apresentaram maior gasto no insumo $\mathrm{S} / \mathrm{M} / \mathrm{V}$, porém, foi o ano com menor gasto no insumo I. No ano 2000, houve maior gasto com S/M/V, embora a eficiência não tenha sido máxima, provavelmente em razão do maior gasto com o insumo MO/ES .

O custo do sal mineral é um componente importante nos custos de manutenção do rebanho de cria em pastagens

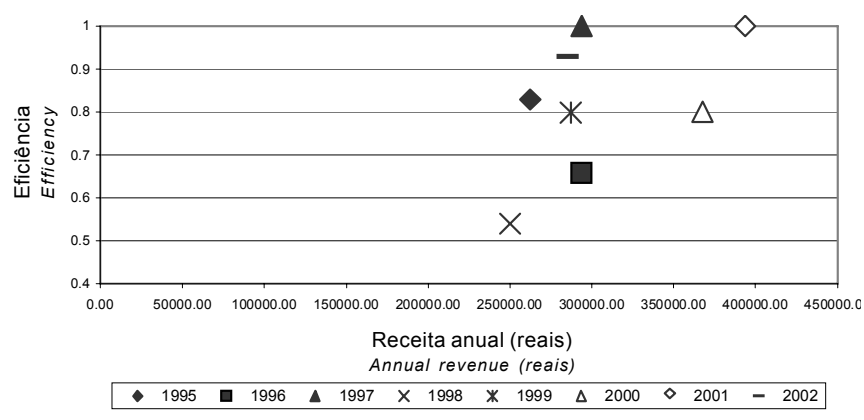

Figura 2 - Eficiência, ao longo dos anos, em relação à receita anual (reais)

Figure 2 - Efficiency, over the years, in relation to annual revenue (reais). 


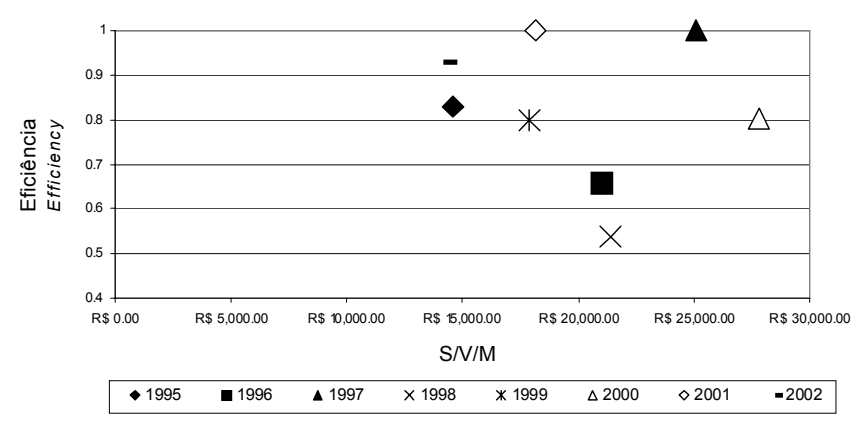

S/V/M - sal mineral/vacinas/medicamentos S/V/M - mineral salt/vaccines/medications.

Figura 3 - Eficiência, ao longo dos anos, em relação ao insumo S/V/M (reais).

Figure 3 - Efficiency, over the years, in relation to the input S/VIM (reais).

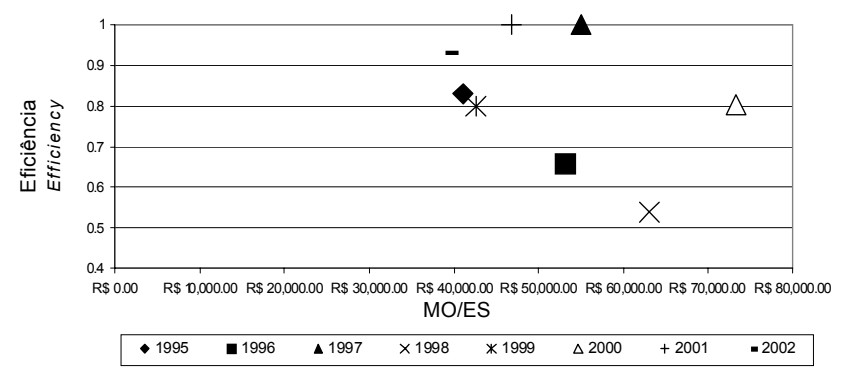

MO/ES - mão-de-obra e encargos sociais.

MO/ES-labor and social security taxes.

Figura 4 - Eficiência, ao longo dos anos, em relação ao insumo $\mathrm{MO} / \mathrm{ES}$ (reais).

Figure 4 - Efficiency, over the years, in relation to the input MO/ES (reais).

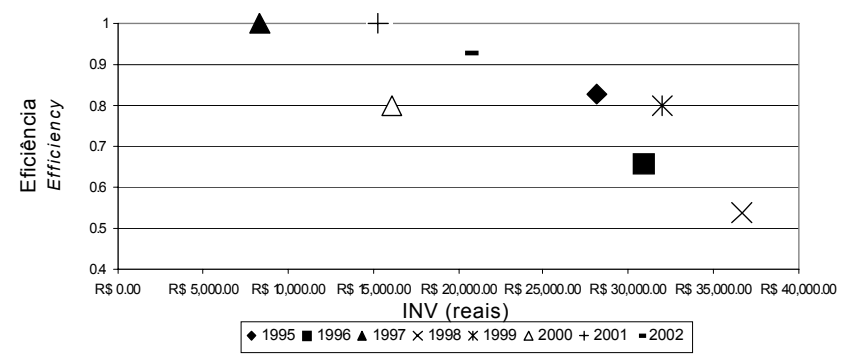

INV - investimentos.

INV - investment.

Figura 5 - Eficiência, ao longo dos anos, em relação ao insumo INV (reais).

Figure 5 - Efficiency, over the years, in relation to the input INV (reais). nativas (Cadavid Garcia, 1986). Entretanto, a utilização desse insumo é básica no Pantanal, como recurso para aumentar a taxa de natalidade e o ganho de peso de novilhas em crescimento (Pott et al., 1987). Portanto, é um insumo essencial ao manejo e à suplementação alimentar do gado de cria.

A mão-de-obra e os encargos sociais foram os insumos de maior percentual na soma de insumos que o produtor utilizou no período analisado. Nos anos de eficiência máxima, os gastos com MO/ES podem ser considerados médios, pois os gastos em 1997 e 2001 estão dentro do intervalo calculado para o período (51.740,86 $\pm 11.884,54$ reais). No Pantanal, a mão-de-obra é sazonal e varia entre anos e dentro de anos, dependendo das características do manejo extensivo do gado de corte na região, baseado nos ciclos das cheias (Barros, 1998). Pode-se inferir que a questão não é o número de empregados para realização do manejo, mas sua experiência na implantação e no desenvolvimento do sistema de produção modificado.

Os anos nos quais ocorreram maiores gastos com investimento foram os de menor eficiência (Figura 5). Porém, de modo geral, os anos nos quais ocorreram maiores investimentos (1996, 1998 e 1999) foram seguidos por anos com maiores eficiências (1997 e 2001).

Poder-se-ia supor que os investimentos realizados estariam prejudicando a eficiência do sistema como um todo. Entretanto, os investimentos realizados forneceram a base física para que as tecnologias fossem implantadas e aprimoradas no sistema de produção.

Outro aspecto importante é que, na introdução de tecnologias em sistemas extensivos de cria no Pantanal, apesar de dinâmico, em decorrência das características ambientais (enchente, qualidade sazonal das pastagens nativas etc), o retorno econômico é lento, tornando necessárias avaliações em longo prazo (Abreu et al., 2001). Portanto, as tecnologias são relacionadas à gestão de conhecimento e de processos, sendo efetivas quando manejadas em conjunto e de forma sistêmica. O importante é investir de forma ordenada para que haja equilíbrio dos gastos em insumos durante os anos analisados.

\section{Conclusões}

A Análise Envoltória de Dados (DEA) é uma ferramenta nova que possibilitou estimar a eficiência de introdução de tecnologias em sistema real de cria extensiva de gado de corte no Pantanal.

A metodologia identificou as fontes das ineficiências em função dos gastos desnecessários com insumos, aspecto fundamental para o produtor na tomada de decisões, além de possibilitar a análise dos anos eficientes em relação aos 
ineficientes, de modo que os eficientes serviram como referência para adoção de novas práticas de manejo zootécnico e de equilíbrio nos gastos com insumos.

\section{Literatura Citada}

ABREU, U.G.P. Análise da adoção de tecnologias em sistema extensivo de criação de gado de corte no Pantanal: um estudo de caso. Viçosa, MG: Universidade Federal de Viçosa, 2004. 134p. Dissertação (Doutorado em Zootecnia) Universidade Federal de Viçosa, 2004.

ABREU, U.G.P.; MORAES, A.S.; SEIDEL, A.F. Tecnologias apropriadas para o desenvolvimento sustentado da bovinocultura de corte no Pantanal. Corumbá: Embrapa Pantanal, 2001. 31p. (Documentos, 24)

ARZUBI, A.; BERBEL, J. Determinación de índices de eficiência mediante DEA em explotaciones lecheras de Buenos Aires. Investigaciones Agrárias: Producción y Sanidad Animales, v. 17, p.103-123, 2002.

AZEVÊDO, J.A.G.; PEREIRA, J.C.; CARNEIRO, P.C.S. et al. Avaliação da divergência nutricional de variedades de cana-deaçúcar (Saccharum spp.). Revista Brasileira de Zootecnia, v.32, n.6, p.1431-1442, 2003.

BANKER, R.; CHARNES, A.; COOPER, W.W. Some models for estimating technical and scale inefficiencies in data envelopment analysis. Management Science, v.30, p.1078-1092, 1984.

BARROS, A.L. Gente pantaneira (Crônicas de sua história) Rio de Janeiro: Lacerda Editores, 1998. 251p.

BLISKA, F.M.M.; GONÇALVES, J.R. Estudo da cadeia produtiva de carne bovina no Brasil. In: CASTRO, A.M.G.; LIMA, S.M.V.; GOERDET, W.J. (Eds.) Cadeias produtivas e sistemas naturais Brasília: Serviço de Produção de Informação, 1998. p.157-183.

BOUSSOFIANE, A.; DYSON, R.G.; THANASSOULIS, E. Applied data envelopment analysis. European Journal of Operational Research, v.52, p.1-15, 1991.

CADAVID GARCIA, E.A. Análise técnico-econômica da pecuária bovina do Pantanal. Sub-regiões da Nhecolândia e dos Paiaguás. Corumbá: EMBRAPA-CPAP, 1986. 92p. (Circular Técnica, 15)

CARDOSO, V.; ROSO, V.M.; SEVERO, J.L.P. et al. Formando lotes uniformes de reprodutores múltiplos e usando-os em acasalamentos dirigidos, em populações Nelore. Revista Brasileira de Zootecnia, v.32, n.4, p.834-842, 2003.

CEZAR, I.M. Racionalização de investimentos em pastagens: uma abordagem sistêmica no processo decisório. In: SIMPÓSIO SOBRE MANEJO DE PASTAGENS, 18., 2001, Piracicaba. Anais... Piracicaba: Fundação de Estudos Agrários Luiz de Queiroz, 2001.p.351-369.

CEZAR, I.M.; SKERRATT, S.; DENT; J.B. Sistema participativo de geração e transferência de tecnologia para pecuaristas: o caso aplicado a Embrapa Gado de Corte. Caderno de Ciência \& Tecnologia, v.17, n. 2, p.135-170, 2000.
CHARNES, A.; COOPER, W.W.; RHODES, E. Measuring the efficiency of decision making units. European Journal of Operational Research, v.2, p.429-444, 1978.

COELLI, T.J. A guide to DEAP version 2.1: a data envelopment analysis program. Armidale: University of New Engaland, 1996 49p. (CEPA Working Papers 08/96).

EL-MAHGARY, S.; LAHDELMA, R. Data envelopment analysis: Visualizing the results. European Journal of Operational Research, v.85, p.700-710, 1995.

EUCLIDES FILHO, K. Produção de bovinos de corte e o trinômio genótipo - ambiente-mercado. Campo Grande: Embrapa Gado de Corte, 2000. 61p. (Documentos, 85)

FRIEDMAN, L.; SINUANY-STERN, Z. Combining ranking scales and selecting variables in the DEA context: The case of industrial branches. Computers and Operation Research, v.25, p.781-791, 1998.

GOMES, A.P. Impactos das transformações da produção de leite no número de produtores e requerimentos de mão de obra e capital. Viçosa, MG: Universidade Federal de Viçosa, 1999. 161p. Dissertação (Doutorado em Economia Rural) Universidade Federal de Viçosa, 1999.

JOHNSON, R.A.; WICHERN, D.W. Applied multivariate statistical analysis. Upper Saddle River: Prentice-Hall, 1999. 816p.

KHATTREE, R; NAIK, D.N. Multivariate data reduction and discrimination with SAS software. Cary: SAS Institute Inc., 2000. $558 \mathrm{p}$.

MCMANUS, C; SAUERESSIG, M.G. Estudo de características lineares de tipo em gado holandês em confinamento total no Distrito Federal. Revista Brasileira de Zootecnia, v.27, n.5, p.906-915, 1998.

PINTO L.F.B.; ALMEIDA, F.Q.; QUIRINO, C.R. et al. Análise multivariada das medidas morfométricas de potros da raça Mangalarga Marchador: análise de componentes principais Revista Brasileira de Zootecnia, v.34, n.2, p.589-599, 2005

POTT, E.B.; BRUM, P.A.R.; ALMEIDA, I.L. et al. Desempenho reprodutivo de bovinos na sub-região dos Paiaguás do Pantanal Mato-grossense. I. Efeito da suplementação mineral e da idade de desmama sobre a idade e o peso ao primeiro parto. Pesquisa Agropecuária Brasileira, v.22, p.1067-1073. 1987.

POTT, E.B.; CATTO, J.B.; BRUM, P.A.R. Períodos críticos de alimentação para bovinos em pastagens nativas, no Pantanal Mato-Grossense. Pesquisa Agropecuária Brasileira, v.24, n.11, p.1427-1432. 1989.

STATISTICAL ANALYSES SYSTEM - SAS. User's guide. version 8.0. Cary: 1999.

TUPY, O; YAMAGUCHI, T. Eficiência e produtividade: conceitos e medição. Agricultura em São Paulo, v.45, n.2, p.39-51, 1998. 